\title{
Overwriting and intrusion in short-term memory
}

\author{
Tyler D. Bancroft $^{1} \cdot$ Jeffery A. Jones $^{1} \cdot$ Tyler M. Ensor $^{1,2} \cdot$ William E. Hockley $^{1}$ • \\ Philip Servos ${ }^{1}$
}

Published online: 4 December 2015

(C) Psychonomic Society, Inc. 2015

\begin{abstract}
Studies of interference in working and short-term memory suggest that irrelevant information may overwrite the contents of memory or intrude into memory. While some previous studies have reported greater interference when irrelevant information is similar to the contents of memory than when it is dissimilar, other studies have reported greater interference for dissimilar distractors than for similar distractors. In the present study, we find the latter effect in a paradigm that uses auditory tones as stimuli. We suggest that the effects of distractor similarity to memory contents are mediated by the type of information held in memory, particularly the complexity or simplicity of information.
\end{abstract}

Keywords Overwriting · Intrusion · Short term memory · Working memory $\cdot$ Interference

The present manuscript is original, has not been published, and is not under consideration elsewhere.

Tyler D. Bancroft banc6110@mylaurier.ca

William E. Hockley

whockley@wlu.ca

Jeffery A. Jones

jjones@wlu.ca

Tyler M. Ensor

tyler.ensor@mun.ca

Philip Servos

pservos@wlu.ca

1 Department of Psychology, Wilfrid Laurier University, 75 University Ave. W., Waterloo, Ontario, Canada N6K 2T8

2 Department of Psychology, Memorial University of Newfoundland, P.O. Box 4200, St. John's, Newfoundland and Labrador, Canada A1C 5S7
Working memory and short-term memory (WM/STM) are vulnerable to interference from irrelevant information. One method used to examine interference is to present subjects with a concurrent processing task during the memory task (e.g., Conway, Kane, \& Engle, 2003; Conway et al., 2005; Klingberg, 1998; Pazzaglia, 1999). Oberauer, Farrell, Jarrold, Pasiecznik, and Greaves (2012) outlined several possible mechanisms through which concurrent processing during WM/STM maintenance can interfere with memory. In one possible mechanism, increased demand on some cognitive resource that is general to both memory maintenance and concurrent processing results in decreased performance (resourcesharing accounts). In another possible mechanism, representations in WM/STM require constant active maintenance and concurrent processing interferes with this task (time-sharing accounts; see the model of Barrouillet, Portrat, \& Camos, 2011). In a third possible mechanism (similarity-based interference), interference occurs between the representations of items held in memory and representations of information processed during the concurrent task.

Evidence for the latter account comes from studies that have found an effect of item-distractor similarity on task performance. Notably, Oberauer et al. (2012a) point out that such effects are not predicted by the first two accounts of interference described above, because these accounts predict that the magnitude of interference is determined by the amount of the shared resource required by the concurrent processing task, or the amount of time dedicated to the concurrent processing task (e.g., Barrouillet et al., 2011). and would apply equally to similar and dissimilar distractor items.

One mechanism that may underlie similarity-based interference is overwriting (e.g., Nairne, 1990; Oberauer \& Kliegl, 2006). In overwriting accounts, item representations in memory are comprised of a set of features. When items in memory share features, they compete for the representations of those 
features in memory, and items can be degraded by "losing" the features to the representation of a different item. As such, distractors would be expected to produce greater interference when they are similar to items in memory, rather than dissimilar. For example, in a study by Lange and Oberauer (2005). subjects held a list of consonant-vowel-consonant trigrams in memory and were asked to read a second set of trigrams aloud during the retention period. Critically, a single trigram from the memory list was selected as a target, and the distractor list was designed to contain a distractor with the same first letter as the target, a distractor with the same second letter as the target, and a distractor with the same third letter as the target. As predicted by overwriting theory, target items were recalled less often than the nontarget items, which did not share features (i.e., letters) with the distractors.

In Lange and Oberauer's (2005) study, a trigram maintained in STM was susceptible to interference when a distractor had the potential to "capture" one of its features (i.e., when a distractor was similar to, rather than dissimilar from, the target trigram). In other words, distractor similarity impaired performance. In contrast, however, a number of studies have reported greater interference between dissimilar distractors and memory items than between similar distractors and memory items. For example, in a common vibrotactile STM task (e.g., Bancroft \& Servos, 2011; Harris, Miniussi, Harris, \& Diamond, 2002; Romo \& Salinas, 2003). subjects are presented with a to-be-remembered vibration to the hand (the target), followed by an unfilled delay, followed by a second vibration (the probe), and are asked to report whether the target and probe are the same vibrational frequency or different vibrational frequencies (or, alternatively, whether the probe is a higher or lower frequency than the target). Bancroft and Servos (2011) presented a brief, irrelevant distractor stimulus during the delay period, with the frequency of the distractor either the same as the target or different from the target. Critically, when the target and probe were different frequencies, the distractor frequency could be the same as the target frequency, shifted toward the probe frequency, or shifted away from the probe frequency. For example, if the frequency of the target was $18 \mathrm{~Hz}$, and the frequency of the probe was $22 \mathrm{~Hz}$, the distractor could be $20 \mathrm{~Hz}$ (in the towardshift condition), $16 \mathrm{~Hz}$ (in the away-shift condition), or $18 \mathrm{~Hz}$ (in the same-as-target condition).

Bancroft and Servos (2011) reported an interference effect, with poorer performance (i.e., a higher proportion of "same" responses) in the toward-shift condition than in the away-shift condition, which was interpreted as evidence that the distractor was encoded into memory and that the probe was being compared against some combination of the target and distractor (or, alternately, that the distractor was replacing the target in memory on some trials). Furthermore, when the target and probe were the same frequency, there was no effect of distractor frequency, with similar performance whether the distractor was offset by $0 \mathrm{~Hz}, 2 \mathrm{~Hz}$, or $4 \mathrm{~Hz}$. This interference effect has been replicated experimentally (Bancroft, Servos, \& Hockley, 2011). and computationally, using both a model of frontal cortex (Bancroft, Hockley, \& Servos, 2013) and a diffusion model (Bancroft, Hockley, \& Servos, 2012b). In the present paper, we will refer to interference that appears to be caused by the encoding of irrelevant information as intrusionbased interference.

Similar interference effects have been found in other modalities. In an early experiment examining short-term memory for simple stimuli, Guilford and Park (1931) employed a paradigm similar to that used by Bancroft et al. (Bancroft \& Servos, 2011; Bancroft et al. 2011a, b). All trials began with subjects lifting a $200 \mathrm{~g}$ target weight, which they compared to a probe that varied in weight from 185 to $215 \mathrm{~g}$. There were three distractor conditions: a "heavier" condition, in which the distractor weighed $400 \mathrm{~g}$; a "lighter" condition, in which the distractor weighed $100 \mathrm{~g}$; and a control condition, in which the distractor weighed the same as the target (i.e., $200 \mathrm{~g}$ ). Performance on the lighter-heavier discrimination task was poorer in the lighter- and heavier-distractor conditions relative to the control condition. Critically, there was evidence that the distractor affected subjects' memory of the target, with more "heavier" responses when the $100 \mathrm{~g}$ distractor was lifted between the target and probe and more "lighter" responses when the $400 \mathrm{~g}$ distractor was lifted between the target and probe.

Mercer and McKeown (2010a) presented a distractor during the delay period of an auditory STM task. Stimuli were complex tones comprised of four harmonics (always including the fundamental and 8 th harmonic). Three types of distractors were used: those whose critical harmonics were shared by the target, those whose critical harmonics were shared by the probe, and novel distractors whose critical harmonics differed from both the target and probe. Compared to performance for distractors including frequencies from the target, performance suffered when the distractor included novel frequencies or frequencies from the probe. Critically, performance for novel distractors exceeded performance for distractors that were similar to the probe. Mercer and McKeown (2010b) reported similar results.

Deutsch (1970) demonstrated that same-different discrimination of pure tones was significantly impaired when six distractor tones were inserted between the target and probe compared to when six spoken digit distractors were inserted. In a subsequent experiment using four tone distractors rather than six, Deutsch (1972) reported that repeating the target among the distractors enhanced discrimination compared to presenting the probe among the distractors. In a recent replication of this experiment, Ries and DiGiovanni (2009) reported poorer discrimination for distractor sequences incorporating the probe relative to distractor sequences incorporating the target. Finally, Deutsch (1973) documented a pattern of results similar to those reported in the vibrotactile domain (Bancroft 
\& Servos, 2011; Bancroft et al. 2011a, b). This time, rather than repeating the target or presenting the probe among the distractors, Deutsch showed that including a distractor differing by a semitone from the target in the opposite direction from the probe (i.e., an away-shift distractor) in the distractor sequence yielded superior performance compared to a condition wherein one of the interpolated distractors was half a semitone different from the target but in the direction of the probe (i.e., a toward-shift). Critically, and again consistent with Bancroft and colleagues' vibrotactile work, although the direction of the shift affected performance on "different" trials (i.e., trials where $f_{\text {target }} \neq f_{\text {probe }}$ ), performance on "same" trials (i.e., $f_{\text {target }}=f_{\text {probe }}$ ) was unaffected by the direction of the shift.

Magnussen, Greenlee, Asplund, and Dyrnes (1991) also found greater interference for dissimilar than for similar distractors. Subjects compared the spatial frequencies of two consecutively presented visual gratings. When a distractor grating was presented during the delay period, the impact on performance increased when the spatial frequency of the distractor was dissimilar to the target.

Why does the similarity of the distractor to items in memory seem to produce different effects in different studies? The answer may lie in the nature of the stimuli used. Most of the above studies have a common attribute that may shed light on the relevant mechanism of interference: They are scalar shortterm memory studies. A growing body of research suggests that short-term memory for scalar stimulus properties (properties of a stimulus that can be represented as a single value, such as auditory pure tone frequency, stimulus duration, or vibrational frequency) is supramodal, relies on a common storage system, and is represented with similar neural codes (e.g., Spitzer \& Blankenburg, 2011, 2012; Spitzer, Fleck, \& Blankenburg, 2014a; see Bancroft, Hockley, \& Servos, 2014b, for a recent review). In this storage system, the salient property of a stimulus (e.g., the frequency of an auditory pure tone) is thought to be represented as a monotonic, often linear or approximately linear function of neuronal firing rates; this coding scheme has been demonstrated or implied for a variety of stimuli, including vibrotactile frequency, auditory frequency, visual flicker frequency, stimulus duration, stimulus amplitude, and the numerosity of a series of brief stimuli (e.g., Bancroft, Hockley, \& Servos, 2012b, 2013, 2014a; Jun et al., 2010; Lemus, Hernández, \& Romo, 2009a, 2009b; Romo, Brody, Hernández, \& Lemus, 1999; Romo \& Salinas, 2003; Spitzer \& Blankenburg, 2012; Spitzer et al., 2014a; Spitzer, Gloel, Schmidt, \& Blankenburg, 2014b; Spitzer, Wacker, \& Blankenburg, 2010).

Although Deutsch's $(1972,1973)$ and Ries and DiGiovanni's (2009) experiments investigating short-term memory for pitch are qualitatively consistent with intrusionbased interference, aspects of these researchers' methodology limit the degree to which this interpretation is valid. First, in all of these studies, four distractors were interpolated between the target and probe. In Deutsch's (1972) and Ries and DiGiovanni's (2009) experiments, one of the conditions repeated the target among the distractors, a second included the probe among the distractors, and a third included neither the target nor the probe among the distractors. Deutsch (1973) took a similar approach, except that critical distractors were displaced by a semitone from the target. In all conditions, the nontarget and nonprobe distractors were drawn randomly from a predetermined frequency range. For an intrusionbased interference interpretation to be valid, we must make the assumption that, because the distractor frequencies were drawn randomly, the distractors would converge on a common frequency value across a sufficient number of trials, producing a neutral effect on performance. However, there is no way of determining whether or not this occurred in the finite number of trials used in Ries and DiGiovanni's and Deutsch's studies.

A second aspect of Ries and DiGiovanni's (2009) and Deutsch's $(1972,1973)$ methodology limits an intrusionbased interference interpretation. In Bancroft et al.'s (Bancroft \& Servos, 2011; Bancroft et al. 2011a, b) vibrotactile experiments, the single distractor was considerably shorter in duration than the target and probe. In spite of this shorter duration, Bancroft et al. still found evidence that it intruded into memory. In contrast, the distractors in Ries and DiGiovanni's and Deutsch's experiments were the same duration as the targets and probes. Consequently, it is possible that, rather than demonstrating limited intrusion into memory, these experiments demonstrated complete overwriting of the target stimulus.

It should be noted that the task used in the present study (and in previous studies, such as those of Bancroft \& Servos, 2011; Bancroft et al. 2011a, b) does not have an active concurrent processing task: Subjects do not actively engage in some active distractor task or activity (e.g., performing mathematical operations, as in certain span tasks; see Conway et al., 2005, for an overview). Rather, the distractor stimulus is irrelevant to the task. Recent evidence suggests that task-irrelevant stimuli (such as distractors) may be inhibited during short-term memory maintenance, resulting in less interference with the contents of memory. Haegens, Osipova, Oostenveld, and Jensen (2010) reported increased alpha-band power over task-irrelevant sensory regions during maintenance compared to the prestimulus interval, and also early in the delay period on correct than incorrect trials, while Haegens, Luther, and Jensen (2012) reported that somatosensory alpha increased to suppress irrelevant information. Critically, increases in alpha activity are thought to be linked to inhibition of neuronal firing (Haegens, Nácher, Luna, Romo, \& Jensen, 2011). Bisley, Zaksas, Droll, and Pasternak (2004) reported that activity in motion-encoding neurons in visual cortex was suppressed during the middle of the delay period but not early or late in the delay. Most 
compellingly, Linke, Vicente-Grabovetsky, and Cusack (2011) applied a decoding method to fMRI data collected during an auditory STM experiment, and found inhibition of activity in auditory cortex during maintenance - specifically, inhibition of activity around the frequency of stored stimuli. While it is theoretically possible that frequency-specific inhibition in sensory cortex plays some role in stimulus comparison or the decision-making process, Lemus et al. (2009a) reported that primary auditory cortex encoded stimulus information during stimulus presentation but did not encode stimulus information during the memory or decision-making processes. Rather, memory and decision-making processes seem to rely on frontal systems (Lemus et al., 2009b).

While scalar STM tasks are of particular use in testing theories of interference, scalar STM also has significant theoretical implications for our understanding of the cognitive and neural structure of working and short-term memory. The recruitment of frontal cortex as the critical storage substrate for scalar STM contrasts with the recruitment of task-relevant sensory cortex as the storage substrate for STM for more complex forms of stimuli (Bancroft et al. 2014a, b, c). While recent theoretical treatments of the neural basis of working and shortterm memory suggest that memory storage relies on the taskrelevant regions of cortex (Postle, 2006). and while recent fMRI studies of WM/STM for relatively complex stimuli that have applied decoding methods have supported this notion (e.g., Christophel, Hebart, \& Haynes, 2012). studies of scalar STM have found information storage in frontal cortex rather than in sensory cortex (Romo \& Salinas, 2003; Spitzer \& Blankenburg, 2012; Spitzer et al., 2014b).

The assumption that scalar stimuli share a common, modality-independent representation implies that the intrusion-based interference effects we have previously identified in vibrotactile STM should also exist in other forms of scalar STM. In the present study, we extend previous vibrotactile interference findings to the auditory domain by adapting the vibrotactile task used by Bancroft and Servos (2011) to use auditory pure tones as stimuli. Specifically, we aim to replicate their intrusion effect in the auditory domainworse performance when the frequency of a distractor is shifted toward that of the probe (the toward-shift condition) than when it is further from the probe than the target (the away-shift condition).

\section{Experiment 1}

\section{Method}

Subjects Twelve undergraduate students from Wilfrid Laurier University participated for course credit. As pointed out in the statistical guidelines for Psychonomic Society journals, the optional stopping rule (e.g., testing for a significant effect as data is collected and stopping once it is found) has been shown to inflate the Type I error rate. We used an ad hoc stopping rule, in which we continued to collect data and retest after a significant effect was found, which has been shown to reduce Type I error relative to the optional stopping rule (Yu, Sprenger, Thomas, \& Dougherty, 2014). In Experiment 1, our effect of interest was the comparison between the different-away and different-toward distractor conditions, in which a significant effect was found at $n=3$, and data collection and confirmatory testing was continued until $n=12$.

Apparatus and materials Auditory stimuli were generated using Audacity (Version 2.0.4) and presented over Sony MDR-NC40 headphones (with the noise-canceling function disabled), using SuperLab (Version 4.5.1, San Pedro, California: Cedrus) running on a Windows XP machine. Target and probe stimuli were pure tones, $1,000 \mathrm{~ms}$ in duration, and all target and probe stimuli had a frequency of either 510 or $515 \mathrm{~Hz}$. Distractor stimuli were $250 \mathrm{~ms}$ in duration and were $505,510,515$, or $520 \mathrm{~Hz}$. Differentfrequency stimuli (both distractor and probe) differed from the target stimulus by $5 \mathrm{~Hz}$. Note that as previous efforts by our group have found little difference between towardshift distractors with frequencies equal to the probe, and toward-shift distractors with frequencies between those of the target and probe (Bancroft \& Servos, 2011; Bancroft et al. 2011a, b). we opted for a relatively large shift in order to maximize statistical power.

Procedure Subjects were presented with 240 trials. On each trial, subjects were presented with a 1,000 ms target stimulus, followed by a $900 \mathrm{~ms}$ unfilled delay, a $250 \mathrm{~ms}$ distractor, a 350 $\mathrm{ms}$ delay, and a 1,000 ms probe stimulus. Stimulus frequencies varied based on a 2 (probe type: same frequency as target vs. different frequency from target) $\times 2$ (distractor frequency: same as target vs. different from target) within-subjects design, with 60 trials in each condition. Critically, on differentprobe/different-distractor trials, the distractor frequency could be shifted away from the target frequency and toward the probe frequency (e.g., $f_{\text {target }}=510 \mathrm{~Hz}, f_{\text {distractor }}=515 \mathrm{~Hz}$, $f_{\text {probe }}=515 \mathrm{~Hz}$ ), or away from the target frequency and also away from the probe frequency (e.g., $f_{\text {target }}=510 \mathrm{~Hz}, f_{\text {distractor }}=$ $505 \mathrm{~Hz}, f_{\text {probe }}=515 \mathrm{~Hz}$ ), with an equal number of trials (30) in each condition.

Subjects were instructed to report whether the target and probe were of the same pitch or different pitches, and to report their decision by pressing $s$ (for "same") or $d$ (for "different") on the keyboard. There was a 1 , $000 \mathrm{~ms}$ delay between subject response and the beginning of the next trial. 


\section{Results and Discussion}

Proportions of correct responses as a function of probe type and distractor type are reported in Table 1, as are proportions of correct responses in the away-shift and toward-shift conditions. Shapiro-Wilk analyses found no violations of normality (all $p \mathrm{~s}>.160$ ).

A 2 (probe type: same-frequency probe vs. differentfrequency probe) $\times 2$ (distractor frequency: same as target frequency vs. different from target frequency) repeatedmeasures ANOVA was performed on the proportions of correct responses. The main effect of probe type approached significance, $F(1,11)=3.813, M S_{e}=.023, p=.077$, partial $\eta^{2}=$ .257 , with performance in the same-probe condition exceeding that in the different-probe condition. No main effect of distractor frequency was found, $F(1,11)=.169, M S_{e}=.004$, $p=.689$, partial $\eta^{2}=.015$. The interaction between probe type and distractor frequency was not significant, $F(1,11)=1.074$, $M S_{e}=.002, p=.322$, partial $\eta^{2}=.089$.

A paired-samples $t$ test was used in a planned comparison between the different probe/different (away-shift) distractor and different probe/different (toward-shift) distractor conditions. Performance on the away-shift condition was significantly better than on the toward-shift condition, $t(11)=$ $5.786, p<.001$. Additional paired-samples $t$ tests found better performance on the different probe/different (away-shift) distractor condition than on the different probe/same-as-target distractor condition, $t(11)=3.649, p=.004$, and better performance on the different probe/same-as-target distractor condition than on the different probe/different (toward-shift) distractor condition, $t(11)=3.039, p=.011$.

This experiment replicated the intrusion effect reported by Bancroft and Servos (2011). with better performance in the away-shift distractor condition than in the toward-shift distractor condition. This finding is consistent with the intrusion of distractors into STM: As the difference between the probe frequency and a combination of target and distractor frequencies is greater in the away-shift condition than in the toward-shift condition, performance is better in the away-shift condition. This effect is further evident in the significant

Table 1 Mean proportions of correct responses from Experiment 1

\begin{tabular}{ll}
\hline Condition & $\begin{array}{l}\text { Mean proportion } \\
\text { of correct } \\
\text { responses (SEM) }\end{array}$ \\
\hline Same probe/same-as-target distractor & $.67(.04)$ \\
Same probe/different distractor & $.64(.03)$ \\
Different probe/same-as-target distractor & $.57(.04)$ \\
Different probe/different (away-shift) distractor & $.67(.04)$ \\
Different probe/different (toward-shift) distractor & $.48(.04)$ \\
Interference effect & .19 \\
\hline
\end{tabular}

differences in performance between the different (awayshift) distractor and the same-as-target distractor conditions and between the same-as-target distractor and different (toward-shift) distractor conditions. Ironically, this intrusion into memory produces an improvement in performance in the away-shift condition. The beneficial effects of the distractor in the away-shift condition should not mask the fact that the distractor is intruding into and interfering with the contents of memory - that the interference has a beneficial effect merely reflects that the information intruding into memory differs from the probe to a greater degree than the original information.

The marginal effect of probe type (with performance better on same-probe than different-probe stimuli) is consistent with a perceptually challenging task. If subjects find it difficult to perceive the difference between two auditory stimuli differing by $5 \mathrm{~Hz}$, they will make a "same" response - in a sense, the "same" response is the default response.

\section{Experiment 2}

In Experiment 1, distractor stimuli could assume frequency values of $505,510,515$, or $520 \mathrm{~Hz}$. Notably, distractors of 510 and $515 \mathrm{~Hz}$ were more common than those of 505 or 520 $\mathrm{Hz}$, as the former were found in both the same- and different/ toward-shift distractor conditions while the latter were found only in the different/away-shift condition. This may have led subjects to deliberately encode the distractor, as doing so would "refresh" memory when distractors had the same frequency as the target. In Experiment 2, we dropped the samefrequency distractor condition, allowing us to equalize the incidence rates of the various distractor frequencies.

\section{Method}

Subjects Twenty-seven undergraduate and graduate students from Wilfrid Laurier University participated for course credit or for a payment of $\$ 8$. Three subjects were excluded from analysis: One subject did not complete the experiment and two subjects performed below chance, leaving a final sample of 24 subjects. All subjects signed a consent form stating that participants had to have normal or corrected-to-normal vision and hearing to participate. Sampling proceeded as in Experiment 1, and our effect of interest again was the comparison between the different-away and different-toward distractor conditions, in which a significant effect was found at $n=20$, and data collection and confirmatory testing was continued until $n=24$.

Apparatus and materials Auditory stimuli were generated using Audacity and presented over Sony MDR-NC40 headphones (with the noise-canceling function disabled), using 
SuperLab (San Pedro, California: Cedrus) running on a Windows 8 machine. Target and probe stimuli were pure tones, $1,000 \mathrm{~ms}$ in duration, and all target and probe stimuli had a frequency of either 510 or $516 \mathrm{~Hz}$. Distractor stimuli were $250 \mathrm{~ms}$ in duration and were 506, 512, 514, or $520 \mathrm{~Hz}$. Different-frequency stimuli (both distractor and probe) differed from the target stimulus by $6 \mathrm{~Hz}$. Distractors always differed from the probe frequency by $\pm 4 \mathrm{~Hz}$; unlike Experiment 1, there was no condition in which the distractor was the same frequency as the target.

Procedure Subjects were presented with 240 trials. On each trial, subjects were presented with a $1,000 \mathrm{~ms}$ target stimulus followed by a $900 \mathrm{~ms}$ unfilled delay, a $250 \mathrm{~ms}$ distractor, a 350 $\mathrm{ms}$ delay, and a 1,000 ms probe stimulus. The target and probe were either the same frequency $\left(f_{\text {probe }}=f_{\text {target }}\right)$ or different frequencies $\left(f_{\text {probe }}=f_{\text {target }} \pm 6 \mathrm{~Hz}\right)$. The distractor frequency differed from the target frequency by $\pm 4 \mathrm{~Hz}$. Critically, in the different-probe condition, the distractor frequency could be shifted away from the target frequency and toward the probe frequency (e.g., $f_{\text {target }}=510 \mathrm{~Hz}, f_{\text {distractor }}=514 \mathrm{~Hz}, f_{\text {probe }}=516$ $\mathrm{Hz}$ ), or away from the target frequency and also away from the probe frequency (e.g., $f_{\text {target }}=510 \mathrm{~Hz}, f_{\text {distractor }}=506 \mathrm{~Hz}$, $f_{\text {probe }}=516 \mathrm{~Hz}$ ). There were 120 trials in the samefrequency probe condition and 60 trials in each of the different-frequency probe conditions.

Subjects were instructed to report whether the target and probe were of the same pitch or different pitches and to report their decision by pressing $s$ (for "same") or $d$ (for "different") on the keyboard. There was a 1,000 ms delay between subject response and the beginning of the next trial.

\section{Results and Discussion}

Proportions of correct responses as a function of probe type and distractor type are reported in Table 2, as are proportions of correct responses in the away-shift and toward-shift conditions. Shapiro-Wilk analyses found violations of normality in the same-probe condition ( $p=.053)$, the different-probe/ away-shift condition $(p=.007)$, and the different-probe/toward-shift condition $(p=.043)$. A planned paired-sample $t$

Table 2 Mean proportions of correct responses from Experiment 2

Condition

Same probe/Different distractor

Different probe/Different (away-shift) distractor

Different probe/Different (toward-shift) distractor

Interference effect
Mean proportion of correct responses $(S E M)$

$.79(.02)$

$.76(.04)$

$.66(.04)$

.09 test found a significant difference in performance between the different-probe/away-shift and different-probe/towardshift conditions, $t(23)=2.864, p=.009$. As the data were nonnormal, we also performed the Wilcoxon signed-rank test, which also found a significant difference between the different-probe/away-shift (median $=.83$ ) and different-probe/toward-shift conditions (median $=.71$ ) $, z=2.716, p=.007, \mathrm{~W}$ $=44$, consistent with the results of the $t$ test.

As in Experiment 1, an effect of probe type was present, with better performance in the same-probe condition (median $=.81)$ than the different-probe condition (median $=.76), t(23)=2.193, p=.039$. The results of the Wilcoxon signed-rank test were consistent with those of the $t$ test, $z=1.963, p=.050, W=73.5$.

The results of Experiment 2 are consistent with those of Experiment 1, with better performance in the different-probe/ away-shift condition than the different-probe/toward-shift condition. The magnitude of the interference effect was smaller in Experiment 2 (.09) than in Experiment 1 (.19), possibly due in part to the smaller distractor frequency shift in Experiment 2 relative to the probe frequency shift in the different-probe condition (4 Hz relative to $6 \mathrm{~Hz}$ ) than in Experiment $1(5 \mathrm{~Hz}$ relative to $5 \mathrm{~Hz})$. The marginally significant effect of probe type found in Experiment 1 was significant in Experiment 2. In general, the results of Experiment 2 replicated those of Experiment 1 and are consistent with the intrusion of distractors into memory.

\section{General Discussion}

The nature of interference in WM/STM is an open and active research topic. A substantial body of research supports overwriting as a mechanism of interference (e.g., Lange \& Oberauer, 2005; Oberauer, 2009; Oberauer \& Lange, 2008; in contrast, though, see Jünger, Kliegl, \& Oberauer, 2014). In the present study, we have extended the evidence for intrusion as a mechanism of interference in short-term memory by replicating the intrusion effect previously found in the vibrotactile modality (Bancroft \& Servos, 2011; Bancroft et al. 2011a, b; Bancroft et al., 2012b, 2013).

Previous studies have demonstrated that distractors can negatively affect same-different auditory frequency discrimination (Deutsch, 1970, 1972, 1973; Ries \& DiGiovanni, 2009). Nevertheless, as discussed above, an intrusion-based interference account of Deutsch's and Ries and DiGiovanni's results is complicated for two methodological reasons: First, the inclusion of multiple distractors during the delay period, only one of which was systematically manipulated relative to the target and probe, and second, the fact that the distractors were equal in duration to the target and probe. The present study avoided both of these limitations by inserting a single, relatively short distractor between the target and probe. 
Consistent with Deutsch's and Ries and DiGiovanni's results, we found better discrimination on trials with away-shift distractors compared to trials with same-frequency and toward-shift distractors and better discrimination for trials with same-frequency distractors compared to trials with toward-shift distractors. To the best of our knowledge, the present study is the first to demonstrate that single brief auditory distractors can intrude into the memory of a substantially longer target.

Scalar STM tasks share common cognitive and neural properties, independent of sensory modality, and have proven to be useful for testing theories of interference, the integration of low-level neural data with higher level imaging and behavioral research, and the development of computational models. In the present study, we have demonstrated that interference effects identified in vibrotactile scalar STM can also be found in auditory scalar STM. Furthermore, we have demonstrated that interference in scalar STM may not appear in the same fashion as overwriting in WM/STM for more complex stimuli due to the simple nature of scalar stimuli.

Why? We propose that the answer has to do with the fact that scalar stimuli may take on a wide range of possible values, whereas complex stimuli may be more restricted - for example, auditory pure tones can take any chosen frequency, while a letter stimulus only has 26 possible values (in English). Consider, for instance, the consonant-vowel-consonant trigrams employed by Lange and Oberauer (2005) in their serial-recall study. Lange and Oberauer's distractors were trigrams sharing features (i.e., single letters) with one of the trigrams in the study list. These stimuli have multiple features. Scalar stimuli, in contrast, have only one relevant dimension, and this dimension may be able to take on a wide range of values. Consequently, integration of the target and distractor (perhaps as weighted averaging; see Bancroft et al., 2012b, 2013) may improve performance, as in the away-shift condition in the present study. This is not the case with stimuli such as letters. (We cannot "average" two letters.) As such, it may be appropriate to consider the present results as a special case of similarity-based interference, where stimuli have a single task-relevant feature (stimulus frequency, duration, amplitude, etc.), but there may be multiple feature units (frontal neurons), each of which can represent the feature.

In one possibility, a target stimulus is held in memory, and a distractor "competes" for the feature units. Some units will encode the distractor, while others will encode the target. In this case, the effects of a distractor on task performance do not come from degradation of the target stimulus, as the target feature can be represented in a single unit (i.e., neuron). Rather, in this case, subjects incorporate the distractor into the decision-making process, possibly due to an inability to select only a single representation in memory for comparison to the probe. In this case, we have intrusion without loss of the original stimulus representation. Note that this differs from interference due to lack of distinctiveness (e.g., Oberauer et al., 2012a) in that interference effects can be produced by distractors that are substantially different from information held in memory. This would seem to make it unlikely that subjects are confusing memory items and distractors.

Another possibility is that competition between target and distractor results in feature units (neurons) encoding some combination of the target and distractor. This is consistent with feature overwriting accounts. The greater effect of dissimilar distractors is due to the limited number of features used to represent simple stimuli in memory. Consider: If there is a relatively large set of available features, then distractors similar to target items will tend to produce greater interference, as they are competing with the target for feature units. In contrast, if we are using very simple stimuli, where there is only one salient feature and items differ in the "value" of that feature, then we would expect less interference for similar distractors (as they are "overwriting" the feature units with a value similar to the original) than for dissimilar distractors (which would "overwrite" the feature units with a different value). The existence of multiple redundant feature units (i.e., frontal neurons) does not change this prediction. In this case, we have intrusion with loss of the original stimulus representation. In the strongest case, the distractor may completely displace the target from memory in some or all trials.

The results of the present study do not allow us to discriminate between these possibilities. What is suggested by the present study, however, is that intrusion into memory may be able to produce interference without requiring overwriting. As in previous studies (e.g., Oberauer et al., 2012a). the interference effects found in the present study cannot be explained by resource-sharing or time-sharing accounts of interference. Presumably, distractors of any frequency require similar amounts of time and resources to process. However, the effects of those distractors differ based on their similarity to target and probe.

While previous research has demonstrated that intrusionbased interference can affect scalar STM (e.g., Bancroft \& Servos, 2011; Bancroft et al. 2011a, b; Bancroft et al., 2012b, 2013). it is less clear what other forms of interference may also operate on scalar STM. This is further complicated by the lack of a clear analogue to the span measure in scalar STM; examinations of interference in WM/STM have often focused on span tasks (e.g., Burgess, Gray, Conway, \& Braver, 2011; Oberauer et al., 2012a; Oberauer, Lewandowsky, Farrell, Jarrold, \& Greaves, 2012). In contrast, we do not yet even have a consistent estimate of scalar STM capacity. Li et al. (2013) estimated a capacity of more than one stimulus for auditory scalar STM, and Bancroft, Hockley, and Servos (2012a) suggested a capacity of more than one stimulus for vibrotactile scalar STM, but later modeling by Bancroft 
et al. (2013) suggested their 2012 results did not conclusively support a capacity of more than one stimulus in the vibrotactile domain.

Many other open questions about scalar STM still exist. For example, are scalar short-term tasks actually working memory tasks, in that they recruit domain-general attentional and/or executive processes (e.g., Engle, 2002; Engle, Tuholski, Laughlin, \& Conway, 1999). Although scalar STM tasks have occasionally been referred to as working memory tasks (e.g., "parametric working memory"; e.g., Romo et al., 1999), and several experimental (Bancroft et al. 2011a, b; Linke et al., 2011) and computational (Bancroft et al., 2013) studies have suggested that inhibitory mechanisms act to protect the contents of scalar STM from interference, we lack unequivocal evidence that they recruit the attentional and/ or executive processes that are characteristic of working memory tasks.

The myriad ways in which interference can appear in shortterm memory tasks is, perhaps, surprising, given the relative simplicity of many such tasks. In the present study, we have described similarity-based interference in a simple auditory short-term memory task. While some previous work (e.g., Lange \& Oberauer, 2005; Oberauer et al., 2012a) has suggested that distractors that are similar to information in memory are more likely to cause interference than dissimilar distractors, the present study builds on previous work suggesting that the opposite pattern can also be found. The relatively direct relationship between memory representations and the activity of the neurons involved in memory maintenance offers an exciting opportunity to study interference in a far deeper manner than has previously been done.

Acknowledgments The present research was supported by NSERC grants to WEH, JAJ, and PS, NSERC scholarships to T. D. B. and T. M. E., and an Ontario Graduate Scholarship to T. D. B. The present manuscript comprises a portion of T. D. B.'s doctoral research.

\section{References}

Bancroft, T. D., Hockley, W. E., \& Servos, P. (2011a). Vibrotactile working memory as a model paradigm for psychology, neuroscience, and computational modeling. Frontiers in Human Neuroscience, 5, 162.

Bancroft, T. D., Hockley, W. E., \& Servos, P. (2012a). Can vibrotactile working memory store multiple items? Neuroscience Letters, 514, $31-34$.

Bancroft, T. D., Hockley, W. E., \& Servos, P. (2012b). Diffusion modeling of interference in vibrotactile working memory. NeuroReport, 23, 255-258.

Bancroft, T. D., Hockley, W. E., \& Servos, P. (2013). Irrelevant sensory stimuli interfere with working memory storage: Evidence from a computational model of prefrontal neurons. Cognitive, Affective, \& Behavioral Neuroscience, 13, 23-34.
Bancroft, T. D., Hockley, W. E., \& Servos, P. (2014a). A shared shortterm memory system for stimulus duration and stimulus frequency. Canadian Journal of Experimental Psychology, 68, 236-241.

Bancroft, T. D., Hockley, W. E., \& Servos, P. (2014b). Does stimulus complexity determine whether working and short-term memory storage relies on prefrontal cortex or sensory cortex? Attention, Perception, \& Psychophysics, 76, 1954-1961.

Bancroft, T. D., Hogeveen, J., Hockley, W. E., \& Servos, P. (2014c). TMS-induced neural noise in sensory cortex interferes with shortterm memory storage in prefrontal cortex. Frontiers in Computational Neuroscience, 8, 23.

Bancroft, T., \& Servos, P. (2011). Distractor frequency influences performance in vibrotactile working memory. Experimental Brain Research, 208, 529-532.

Bancroft, T. D., Servos, P., \& Hockley, W. E. (2011b). Mechanisms of interference in vibrotactile working memory. PLOS ONE, 6, e22518.

Barrouillet, P., Portrat, S., \& Camos, V. (2011). On the law relating processing to storage in working memory. Psychological Review, 118, 175-192.

Bisley, J. W., Zaksas, D., Droll, J. A., \& Pasternak, T. (2004). Activity of neurons in cortical area MT during a memory for motion task. Journal of Neurophysiology, 91, 286-300.

Burgess, G. C., Gray, J. R., Conway, A. R. A., \& Braver, T. S. (2011). Neural mechanisms of interference control underlie the relationship between fluid intelligence and working memory span. Journal of Experimental Psychology: General, 140, 674-692.

Christophel, T. B., Hebart, M. N., \& Haynes, J.-D. (2012). Decoding the contents of visual short-term memory from human visual and parietal cortex. Journal of Neuroscience, 32, 12983-12989.

Conway, A. R. A., Kane, M. J., Bunting, M. F., Hambrick, D. Z., Wilhelm, O., \& Engle, R. W. (2005). Working memory span tasks: A methodological review and user's guide. Psychonomic Bulletin \& Review, 12, 769-786.

Conway, A. R. A., Kane, M. J., \& Engle, R. W. (2003). Working memory capacity and its relation to general intelligence. Trends in Cognitive Sciences, 7, 547-552.

Deutsch, D. (1970). Tones and numbers: Specificity of interference in immediate memory. Science, 168, 1604-1605.

Deutsch, D. (1972). Effect of repetition of standard and of comparison tones on recognition memory for pitch. Journal of Experimental Psychology, 93, 156-162.

Deutsch, D. (1973). Interference in memory between tones adjacent in the musical scale. Journal of Experimental Psychology, 100, 228-231.

Engle, R. W. (2002). Working memory capacity as executive attention. Current Directions in Psychological Science, 11, 19-23.

Engle, R. W., Tuholski, S. W., Laughlin, J. E., \& Conway, A. R. A. (1999). Working memory, short-term memory, and general fluid intelligence: A latent-variable approach. Journal of Experimental Psychology: General, 128, 309-331.

Guilford, J. P., \& Park, D. G. (1931). The effect of interpolated weights upon comparative judgments. American Journal of Psychology, 43, 589-599.

Haegens, S., Luther, L., \& Jensen, O. (2012). Somatosensory anticipatory alpha activity increases to suppress distracting input. Journal of Cognitive Neuroscience, 24, 677-685.

Haegens, S., Nácher, V., Luna, R., Romo, R., \& Jensen, O. (2011). $\alpha$ Oscillations in the monkey sensorimotor network influence discrimination performance by rhythmical inhibition of neuronal spiking. Proceedings of the National Academy of Sciences, 108, 1937719382.

Haegens, S., Osipova, D., Oostenveld, R., \& Jensen, O. (2010). Somatosensory working memory performance in humans depends on both engagement and disengagement of regions in a distributed network. Human Brain Mapping, 31, 26-35. 
Harris, J. A., Miniussi, C., Harris, I. M., \& Diamond, M. E. (2002). Transient storage of a tactile memory trace in primary somatosensory cortex. Journal of Neuroscience, 22, 8720-8725.

Jun, J. K., Miller, P., Hernández, A., Zainos, A., Lemus, L., Brody, C. D., \& Romo, R. (2010). Heterogenous population coding of a shortterm memory and decision task. Journal of Neuroscience, 30 , 916-929.

Jünger, E., Kliegl, R., \& Oberauer, K. (2014). No evidence for feature overwriting in visual working memory. Memory, 22, 374-389.

Klingberg, T. (1998). Concurrent performance of two working memory tasks: Potential mechanisms of interference. Cerebral Cortex, 8 , 593-601.

Lange, E. B., \& Oberauer, K. (2005). Overwriting of phonemic features in serial recall. Memory, 13, 333-339.

Lemus, L., Hernández, A., \& Romo, R. (2009a). Neural codes for perceptual discrimination of acoustic flutter in the primate auditory cortex. Proceedings of the National Academy of Sciences, 106, 9471-9476.

Lemus, L., Hernández, A., \& Romo, R. (2009b). Neural encoding of auditory discrimination in ventral premotor cortex. Proceedings of the National Academy of Sciences, 106, 14640-14645.

Li, D., Cowan, N., \& Saults, J. S. (2013). Estimating working memory capacity for lists of nonverbal sounds. Attention, Perception, \& Psychophysics, 75, 145-160.

Linke, A. C., Vicente-Grabovetsky, A., \& Cusack, R. (2011). Stimulusspecific suppression preserves information in auditory short-term memory. Proceedings of the National Academy of Sciences, 108, 12961-12966.

Magnussen, S., Greenlee, M. W., Asplund, R., \& Dyrnes, S. (1991). Stimulus-specific mechanisms of visual short-term memory. Vision Research, 31, 1213-1219.

Mercer, T., \& McKeown, D. (2010a). Interference in short-term auditory memory. Quarterly Journal of Experimental Psychology, 63, 12561265.

Mercer, T., \& McKeown, D. (2010b). Updating and feature overwriting in short-term memory for timbre. Attention, Perception, \& Psychophysics, 72, 2289-2303.

Nairne, J. S. (1990). A feature model of immediate memory. Memory \& Cognition, 18, 251-269.

Oberauer, K. (2009). Interference between storage and processing in working memory: Feature overwriting, not similarity-based competition. Memory \& Cognition, 37, 346-357.

Oberauer, K., Farrell, S., Jarrold, C., Pasiecznik, K., \& Greaves, M. (2012a). Interference between maintenance and processing in working memory: The effect of item-distractor similarity in complex span. Journal of Experimental Psychology: Learning, Memory and Cognition, 38, 665-685.

Oberauer, K., \& Kliegl, R. (2006). A formal model of capacity limits in working memory. Journal of Memory \& Language, 55, 601-626.

Oberauer, K., \& Lange, E. B. (2008). Interference in verbal working memory: Distinguishing similarity-based confusion, feature overwriting, and feature migration. Journal of Memory and Language, $58,730-745$

Oberauer, K., Lewandowsky, S., Farrell, S., Jarrold, C., \& Greaves, M. (2012b). Modeling working memory: An interference model of complex span. Psychonomic Bulletin \& Review, 19, 779-819.

Pazzaglia, F. (1999). The role of distinct components of visuo-spatial working memory in the processing of texts. Memory, 7, 19-41.

Postle, B. R. (2006). Working memory as an emergent property of the mind and brain. Neuroscience, 139, 23-38.

Ries, D. T., \& DiGiovanni, J. J. (2009). Effects of recurrent tonal information on auditory working memory for pitch. Hearing Research, 255, 14-21.

Romo, R., Brody, C. D., Hernández, A., \& Lemus, L. (1999). Neuronal correlates of parametric working memory in the prefrontal cortex. Nature, 399, 470-473.

Romo, R., \& Salinas, E. (2003). Flutter discrimination: Neural codes, perception, memory and decision making. Nature Reviews Neuroscience, 4, 203-218.

Spitzer, B., \& Blankenburg, F. (2011). Stimulus-dependent EEG activity reflects internal updating of tactile working memory in humans. Proceedings of the National Academy of Sciences of the United States of America, 108, 8444-8449.

Spitzer, B., \& Blankenburg, F. (2012). Supramodal parametric working memory processing in humans. Journal of Neuroscience, 32, $3287-$ 3295.

Spitzer, B., Fleck, S., \& Blankenburg, F. (2014a). Parametric alpha- and beta-band signatures of supramodal numerosity information in human working memory. Journal of Neuroscience, 34, 4293-4302.

Spitzer, B., Gloel, M., Schmidt, T. T., \& Blankenburg, F. (2014b). Working memory coding of analog stimulus properties in the human prefrontal cortex. Cerebral Cortex, 24, 2229-2236.

Spitzer, B., Wacker, E., \& Blankenburg, F. (2010). Oscillatory correlates of vibrotactile frequency processing in human working memory. Journal of Neuroscience, 30, 4496-4502.

Yu, E. C., Sprenger, A. M., Thomas, R. P., \& Dougherty, M. R. (2014). When decision heuristics and science collide. Psychonomic Bulletin \& Review, 21, 268-282. 